\title{
Historical Background of the Translatological Dictionaries
}

\author{
Lei Gao \\ School of Foreign Languages, Huaiyin Normal University, Huai'an, Jiangsu, China \\ E-mail: mrgaolei@163.com
}

\begin{abstract}
This paper will first talk about the history, production, and importance of the dictionaries. Dictionaries play an important role in developing the social culture so it is quite necessary and reasonable to take them as a kind of research subject. Lexicography, which is generally considered to be built on modern linguistics, must possess its own theoretical system and research methodology. In terms of the properties of lexicography, the paper will discuss such aspects as multidisciplinary integration, open perspectives, and practice orientation. Any new perspective to deal with the dictionary may be possible or feasible on condition that it satisfies the requirements of lexicography. With the development of translatology and lexicography, the translatological dictionaries (TD) came into being. The TD has already become a research subject under translatology in China.
\end{abstract}

Index Terms - dictionary, lexicography, translatology, translatological dictionaries (TD)

\section{INTRODUCTION}

To trace the origin of the TD, this chapter will first survey the development and some properties of the dictionary. There is a long history of the dictionary culture, but lexicography, which mainly deals with the dictionary-making and dictionary research, is just developed in the latter half of the $20^{\text {th }}$ century. The discussion of the dictionary, lexicography, and their properties or characteristics will reveal the dictionary tradition of the TD. The naissance of the TD comes from the integration of lexicography and translatology.

\section{DICTIONARY}

With the cultural development, it is necessary for people to store, spread or normalize the various kinds of knowledge, so dictionaries are produced. The dictionary is a kind of cultural crystallization, without which it is almost incredible for a certain civilization to survive. The dictionary, to some extent, can reflect its corresponding cultural conditions. Judging from the history, we find that any mature academic discipline must have its corresponding dictionaries. We can safely say that the dictionary-making and research is a very important cultural activity. YONG He-ming said:

From relevant literature, we can conclude that the earliest dictionary originated from the Middle East. The dictionary-making has a history of more than 4000 years, which can be dated back to 5000 years BC in the southern region of Mesopotamia, where Sumerian was spoken in the tribes. (YONG He-ming 2006: 27)

In China, the dictionary culture has a history of about 2200 years, which can be traced back to the dictionary of $E r$ $Y a$ (《尔雅》). In Europe, the earliest Greek glossaries, the preliminary dictionaries, were mostly used to explain the difficult words in a particular author's work, especially to help understand the canons of the $8^{\text {th }}$ century BC, e.g. Homer's Iliad and Odyssey. In India, in the 1000 years BC or so, the old Indians listed out some difficult words in the Buddhist scripture of Veda, and made a pamphlet of the glossary to help spread the scripture, which is regarded as the start of the dictionary-making in India. The compilation of dictionaries is an inevitable outcome of the development of culture and language, and any dictionary is a marked achievement in developing a certain culture.

The dictionary history of China, according to the statement of YONG He-ming, can be divided into four phases: 1) Budding and initiate phase (from the West Zhou to Han dynasties); 2) Exploring and building phase (from Wei and Jin dynasties to Yuan dynasty); 3) Evolution and shaping phase (from Ming dynasty to Qing dynasty); 4) Dreary and thriving phase (during $20^{\text {th }}$ century). (YONG He-ming 2006: 8) In the past, the theories about dictionary-making were mainly composed of the comments of a particular dictionary, and they appeared more often than not as the prefaces, notes on the use, appendices of the dictionaries, and so on. During the $20^{\text {th }}$ century, with the introduction of modern linguistics and other related theories, the compilation techniques of dictionaries were getting more advanced, and the theoretical system of lexicography had been set up.

In modern society, dictionaries and other reference books are playing a very important role, as they are greatly conducive to people's practice and research. We can even say that excellent dictionaries in a certain field represent, to a great extent, the maturity of the field. In this respect, SUN Ying-chun remarked:

The dictionary is a kind of tool used to learn knowledge and go in for research. The importance of the dictionary is salient in the information society. In some developed countries, dictionaries occupy more than $20 \%$ of all the 
publications, so the dictionary culture is formed. The study of dictionary is becoming more and more important, and it has branched off from linguistics, standing on its own firm feet as an independent discipline. (SUN Ying-chun 2008: Preface)

\section{A. Definition of the Dictionary}

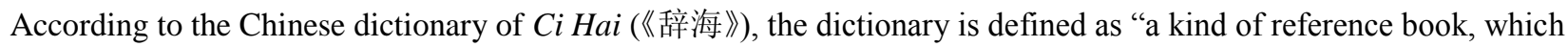
collects the vocabulary of a particular language, arranges them in a certain order, and gives definitions to each one." The definition offered by the Collins Cobuild's Essential English Dictionary (CCEED) is a little more detailed: 1) A book in which the words of a language are listed alphabetically and their meanings are explained. 2) A book in which words in one language are listed alphabetically and are followed by the words in another language which have the same meaning. 3) An alphabetically ordered reference book on a particular subject. The definition of the CCEED covers three kinds of dictionaries: general dictionary; bilingual dictionary; specialized dictionary. Though comparatively complete in dealing with the types of dictionaries, this definition is still loose in nature, as it does not pinpoint all the noumenal characteristics of a dictionary. It is perhaps very tricky to give a perfect or ideal definition of a dictionary, as different lexicographers may stress different aspects of a dictionary.

HUANG Jian-hua argued that "the dictionary is a kind of reference book, in which the lemmas are collected in a certain order and dealt with separately to supply a certain quantity of information." (HUANG Jian-hua 2001: 2) We think that HUANG Jian-hua's definition of the dictionary is more accurate, as it touches the form, content and function of the dictionary. As far as the form is concerned, this definition stresses that the entries (words) should be conducted respectively as independent texts, and the entries should be arranged according to a kind of order, which is conducive to being consulted; as far as the content is concerned, a dictionary should centre on words or vocabulary, and the range and scale the entries cover in information should be determined by the nature and scale of the dictionary. In terms of the nature of a dictionary, LI Er-gang put forward:

A dictionary is a kind of miniature linguistic work, which collects the words (vocabulary) to solve the puzzles, and in which the entries are dealt with separately, and supplied with definitions and relevant knowledge. (LI Er-gang 2002: 13)

LI Er-gang's definition gets the characteristics of the entry, but he thinks that a dictionary acts as a kind of linguistic work, which is meant to spread linguistic knowledge instead of being consulted as a kind of reference book, and thus the function of a dictionary is a little misunderstood. In the article of "Characteristics and Entry-Explaining Principles of Translatological Dictionaries", ZHANG Bo-ran argued that a dictionary should be composed of two elements, which means that:

One element lies in the form; the other element lies in the function. A dictionary must have the headwords and their definitions, which are indispensable to any dictionary, the headwords lexicographically called "left core" and their definitions "right core". This is the formal elements. The main task of the dictionary is to pertinently offer readers information and solve their puzzles. This is the function of the dictionary, which constitutes their functional element. The formal function and the functional function are mutually connected and unified. (See SUN Ying-chun 2005: 4)

ZHANG Bo-ran's definition stresses the formal and functional elements of the dictionary. His theory is reasonable, for it is perhaps just the formal and functional elements that determine the being of a dictionary.

From the aforementioned statements about the definition of the dictionary, we can find that the form, content and function are the most important elements in determining the being of the dictionary. Integrating the nature, function, stylistic layout and so on of the dictionary, SUN Ying-chun stated:

The dictionary is a kind of reference book which collects words, deals with the words respectively and organizes them in a certain manner. By offering a certain quantity of information, this kind of reference book can describe the language, regulate the application of the language, solve people's puzzles and promote human communication. (SUN Ying-chun 2008: 114)

In addition, there are many other definitions about the dictionary, of which "the best definition in metalexicography is actually a list of features draw up by Rey-Debove for her study of modern French dictionaries." (Béjoint 2002: 9) According to Rey-Debove, a dictionary can be defined by the following eight characteristics:

a) A dictionary is a series of separate paragraphs;

b) A dictionary is meant to be consulted, not read;

c) Dictionaries have a double structure;

d) A dictionary is an ordered set;

e) A dictionary is a list of linguistic units;

f) A dictionary is a didactic book;

g) A dictionary gives information about linguistic signs;

h) A dictionary is a structured representation of predetermined lexical set.

(Béjoint 2002: 10-24)

From these characteristics, we can find that a), c) and d) stress the formal elements of the dictionary; b) and f) stress the functional elements; e), g) and h) stress the contents. Therefore, we can say any definition of the dictionary should centre on the formal, functional and content elements, which in turn constitute the criteria to measure a dictionary. Besides, the formal elements of a dictionary mainly consist of the lemmas and their definitions, the former 
lexicographically called "left core" and latter "right core". And the main task of a dictionary is to offer users information and solve their problems, which is called the reference function of a dictionary. The formal element and the functional element should be unified organically.

\section{B. Properties of the Dictionary}

The properties of a dictionary can reflect its nature, so different kinds of dictionaries may possess different properties. Understanding the properties, we can better make clear the nature and function of a dictionary. In addition, there exist properties that almost all the dictionaries may share in common.

The prescriptiveness and descriptiveness of the dictionary remain as a constant topic for the lexicographers. Generally speaking, dictionaries have two main functions, one to record the situation about a language and its culture and the other to normalize a language. The former function is descriptive in nature, and the latter prescriptive. With the development of the dictionary-making, not all the dictionaries can equally show both these properties, some to be descriptive and some to be prescriptive. Béjoint wrote:

The aim of the very first monolingual dictionaries was to describe lexical usage, but in the eighteenth century lexicographers felt it was their duty to indicate - and in some cases decide-what was good and what was bad usage. They recommended some forms and banned others. (Béjoint 2002: 100)

Rey-Debove once argued that the prescriptiveness and descriptiveness are based on two different norms: a "qualitative" norm and a "quantitative" norm (Rey-Debove 1972). In terms of these two norms, Béjoint wrote:

The qualitative norm is based on the usage and on the opinion of the "best" language users, as determined by a more or less clear consensus - often, in fact, only educators and well-known writers. (Béjoint 2002: 100)

The quantitative norm is based on the observation of the linguistic usage of all the reasonably fluent users of the community. Any form is good as long as it is used by a certain number of speakers. The difficulty here is to determine the minimum number of speakers, or of recorded uses, required to make a usage acceptable. The quantitative norm corresponds to the modern corpus, which can be studies with statistical methods in order to determine frequencies. The dictionary is prescriptive if it uses a qualitative norm, descriptive if it uses the quantitative. (ibid)

From Béjoint's statement, we know that the prescriptive dictionary is to normalize a language and the descriptive dictionary is to faithfully record the use situation of a language. Zgusta once remarked, "The aim of prescriptive dictionaries is to fix the language, to try to prevent it from changing, change usually being equated with deterioration." (ibid) Being afraid of the deterioration of a language, lexicographers tend to design prescriptive dictionaries. People may think that all the dictionaries are prescriptive, as any dictionary can give an authoritative impression upon its readers. In addition, a dictionary is always a reference book, in which one tries to find out the relevant information to solve their puzzles, so it would seem more normative than descriptive. But more often than not,

The distinction between descriptive dictionaries and prescriptive dictionaries is much clearer in the declarations of lexicographers and of some of their public than in the reality of the dictionaries themselves. Total descriptiveness is impossible, because the lexicographer can not avoid making choices, and total prescriptiveness does not work if it is estranged from the realities of usage ... (Béjoint 2002: 101)

The macrostructure and microstructure of the dictionary constitute the text of a dictionary. The structure of a dictionary, which can be compared to the muscular tissues or vessels of a dictionary, is the carrier of the compilation ideas about a particular dictionary. Traditionally, the structure of a dictionary is considered to consist of the macrostructure and microstructure, which in turn consist of other substructures. Macrostructure usually refers to "the overall List structure which allows the compiler and the users to locate information in a Reference Work". (Hartmann \& James 2000: 91) Microstructure usually refers to the detailed information about the lemmas. Macrostructures mainly deal with vertically the arrangements of entries, which are mostly based on nouns (or terms, concepts); microstructures mainly deal with horizontally the arrangements of the information about a lemma. The interweavement of the macrostructure and microstructure will make up a systematic textural structure of a dictionary.

Dictionaries vary according to the amount of information they provide and how they present the information. Different types of dictionaries may require different arrangements of the macrostructures and microstructures. And the contents of these two structures are closely related to the lexicographers' purposes. The lemmas, which are usually based on nouns (or terms, concepts), should be concise, typical and systematic in presenting the related materials. The right core of a lemma should systematically arrange the information the designers want to present to the prospective users. In addition, the number of lemmas is often subject to the scale of a dictionary. Moreover, Fenner (1998) claimed that dictionaries are independent texts since their entries meet all the normal criteria of referential cohesion. (Hartmann 2005: 63)

The structure of something is the way in which it is made, built, or organized; a structure is something that has been built or formed in a particular way; a system or activity that has structure is well arranged and organized and is therefore efficient (CCEED 1989). From this definition, we can find that a structure should be systematic, watertight, and efficient in its organization. As for the "structure" of the dictionary, Hartmann pointed out that, in the literature on lexicography, it had been used in a similar fashion to refer to the manner in which the component parts of a dictionary are related to each other and to the whole. (Hartmann 2005: 57-58)

In addition, LI Er-gang proposed that dictionaries should have such properties as: the words collected for the users' reference; comprehensive definitions; intensive knowledge; separate retrieval. (Li Ergang 2002: 4) Collecting the words 
for the users' reference is the purpose of the lexicographers, as dictionaries are used to be consulted rather than to be read. Comprehensive definitions mean that the language used in a dictionary is either to define or to be defined. Intensive knowledge means that it should be intensive in presenting the related knowledge. Separate retrieval, a formal property of the dictionary, means that the dictionary unit should lie in the individual lemma that is relatively independent; meanwhile, the lemmas should be in an aggregate arrangement, which can form a system to convenience the readers' reference.

\section{Function of the Dictionary}

Dictionaries are generally considered as a kind of reference book, in which people can consult the relevant information they want. This can be called the dictionary's function of practicality, which is generally regarded as a criterion to evaluate the quality of the dictionaries. For a long time, the dictionaries have been endowed with such images as guardian of the purity of language, as thesaurus of the whole lexicon, as thesaurus of all the collective knowledge of the society, as guardian of absolute and eternal truth, as guardian of the moral and ideological values of the society, as a means of social promotion, and as a patriotic emblem. (Béjoint 2002: 116-138) Some of these images are derived from the laymen's perception, and some from lexicographers' empiricism.

The function of dictionaries should be closely related to their types. In this respect, J. et C. Dubois argued that dictionaries should realize such functions:

1) Dictionaries can help the readers to understand, translate and write another language, or to communicate with the people speaking another language, which constitutes the functions of the bilingual or multilingual dictionaries;

2) Dictionaries can help the readers to understand the special terms or the vocabularies about science and technology on the basis of their mastered general words, which constitutes the functions of the various specialized dictionaries;

3) Dictionaries can help the readers to well master the linguistic expressions and to improve their philological knowledge by offering related semantic, syntactic, morphological and phonetic information, which constitutes the functions of the monolingual language dictionaries;

4) Dictionaries can help the readers to extend their knowledge or to deepen their knowledge by offering various kinds of knowledge in the entries, which constitutes the functions of the various encyclopedic dictionaries. (HUANG Jian-hua 2001: 5-6)

The quality of dictionaries is determined, to a certain extent, by the functions accomplished in the users. To realize the various functions of the dictionary is mainly preconditioned by the scientificalness, knowledgeableness, practicality, and so on of the dictionary. Nowadays "the dictionary continues to be seen as an instrument designed to provide quick and superficial support in case of emergency." (Béjoint 2002: 152) Trying to serve the users, the dictionary should try to satisfy the preset objective of compilation. But "research into dictionary use has revealed that there can be a discrepancy between the functions intended by the compiler and the actual look-up practices in specific situations of use." (Hartmann \& James 2000: 60)

YONG He-ming argued:

The traditional study of the function of dictionaries has always been influenced by the research method of the linguistic noumenon, so it can not get out from the confinement of the instrumental theory, which takes the dictionary as a kind of reference book to be consulted. (YONG He-ming 2006: 194)

We know that the function of the dictionary is more than to be a reference book, as the dictionary is sure to be endowed with the functions of communication, culturing, ideological instruction and the like. So YONG He-ming generalized three functions of the dictionary: "descriptive function, didactic function, ideological function". (YONG He-ming 2006: 194) In terms of the functions of the dictionary, the statement made by SUN Ying-chun is very inspiring. He said:

The dictionary is product of the social needs, and it is like a mirror of the society, reflecting the social development. In modern society, with the increasingly detailed division of social labor, the dictionary today is incomparable in the quantity, quality, variety, and so on. The knowledge and information people need to consult are becoming more than those that people need to read or memorize. Generally speaking, the role the dictionary plays in the society is becoming more and more salient, and greater and greater. (SUN Ying-chun 2008: 5)

\section{LEXICOGRAPHY}

\section{A. Development of Lexicography}

For a long time, people have issued their research achievements, comments, ideas or suggestions on the nature, properties, types, functions and some other aspects of the dictionary. Thus, with the accumulation of the achievements made in the dictionary making and research, lexicography has wormed its way to an independent discipline. Nowadays, as an independent discipline, lexicography has formed its theoretical system, research methodology, representative works, leading figures, and so on. To study its course of development can give us much inspiration.

The dictionary-making can be dated back to more than 4000 years ago, so we can say that lexicography has a glorious history. Meanwhile, we can also say that lexicography is very young, for lexicography should be based on modern linguistics, and its theoretical system and research methods had not formed until the contemporary times. In the past, the compilation of dictionaries was just taken as empirical practice without any academic value. "Lexicographers 
have always been linguists of a sort, but they have tended to be considered as non-linguists, and to be rejected by the academic world of linguistics." (Béjoint 2002: 169) So for a long time, the dictionary had been considered to be too unscientific to be worthy of academic concern, and only an impure by-product of linguistics. At the same time, dictionary-makers thought that the compilation of dictionaries was a kind intuitional work, which had nothing to do with theories. In this respect, Henri Béjoint wrote:

Lexicographers and dictionary publishers did not particularly want the contribution of linguists in the compilation of dictionaries either. They failed to see what linguists could contribute to the practical task of dictionary-making: they thought that academics would be of little use in lexicographical work, with all its practical and social constraints, to which linguistic theory is ill-adapted. (Béjoint 2002: 170)

As for the early dictionary-making, YONG He-ming also described:

The early dictionary-makers hardly had any guidance from the linguistic theories, and knew nothing about the application of the phonetic knowledge. They solved the problems about dictionary-making only by their acute insight and subjective perception, surmising the meaning of the words and their approximate pronunciation, so mistakes were unavoidable. The early glossaries and dictionaries were characterized by their comprehensive lemmas, monotonous functions, poor information, and messy structures. (YONG He-ming 2006: 37)

Dictionary compilation is a part of cultural activities, leaving behind a long history. In this respect, Hartmann remarked, "In Europe, the lexicographic tradition goes back to early Greek glossaries in the fifth pre-Christian century, in Mesopotamia, southern India and China even further." (Hartmann 2005: 6) From these early glossaries, we can find the prototype of modern dictionaries and their functions. But the first actual English dictionary did not appear until the $17^{\text {th }}$ century. For a long time in the past, the dictionary making had escaped from the academic research. In the $18^{\text {th }}$ century in the West, lexicographers began to talk about the quality of dictionaries, and "it is reported that Albert Way was the first scholar to study the compilation of English dictionaries. His research subject was The Promptorium Parvulorum Sive Cleric rum, an English-Latin bilingual dictionary, which can be dated back to 1843." (YONG He-ming 2006: 83) The case in China is much later, as is pointed out by LI Er-gang. He remarked:

In China, there was almost vacant in dictionary theories before 1970s, and therefore, there was no talk about the characteristics of dictionaries. In 1979, Ci Hai, a grand comprehensive dictionary, was republished, which embodied the painstaking effort and the wisdom of a new-generation scholars. In summing up their experience of compilation, scholars began to generalize the principle knowledge about dictionaries. (LI Er-gang 2002: 1)

What LI Er-gang said is not completely correct, as we can easily find there is a lot of literature before 1970s about the dictionary compilation, which always appeared in the preface, postscript, appendices or stylistic notes of the dictionaries. Maybe we can say that modern dictionary theories did not come into being until 1970s, for they must be based on modern linguistics.

YONG He-ming argued, "The compilation of dictionaries originated directly or indirectly from relevant language studies, the achievements of which were completely or partially employed in the dictionary-making." (Yong He-ming 2006: 80) In 1898, the publication of MA Jian-zhong's Ma Shi Wen Tong (《马氏文通》) marked the naissance of Chinese linguistics. After that, YONG He-ming pointed out:

Grammar, lexics, phonetics, semantics, dialectology and the other research achievements of the branched studies of linguistics were increasingly used in the practice of dictionary-making and theoretical studies, which had set the foundation for the development of the lexicographical theories. (YONG He-ming 2006: 573)

From the forties of the $20^{\text {th }}$ century, the linguists and lexicographers began to show interest in each other's profession. In this respect, Béjoint described as follows:

The many conferences on lexicography are attended by linguists as well as lexicographers: the first was held in Bloomington, Indiana, in 1960, and it was followed by many others. The first to be held on English territory was organized by R. Hartmann in 1979 at Exeter. Several well-known linguists (William Labov, Uriel Weinreich, James McCawley, Charles Fillmore, Dwight Bolinger, Randolph Quirk, Anna Wierzbicka, etc.) have published papers or books on dictionaries. Some have even been closely involved in the compilation of dictionaries: David Crystal, A. C. Gimson, John Sinclair, Igor Mel'čuk, etc. At the same time, many practicing lexicographers are also expert metalexicographers or linguists: Patrick Hanks, Robert Ilson, etc. (Béjoint 2002: 172)

Lexicography and linguistics are now inextricably mixed. With the help of linguistics, lexicographers have come to get a deep knowledge of the content, function, form and the other aspects of the dictionary, which have developed into lexicographical theories. In the latter half of the $20^{\text {th }}$ century, lexicography witnessed its great progress. Modern linguistics and many other disciplines gave lexicography a great momentum of development. In addition to many kinds of dictionaries and academic papers about dictionary, several specialized journals of lexicography were launched, a large number of specialized conferences organized, and some special series of metalexicographical books were published.

YONG He-ming pointed out that "the early lexicographical studies is mainly confined to the partial and micro-level studies of the dictionary, e.g. the compilation skills, a certain section of the compilation process, or the comments or studies of a particular dictionary." (YONG He-ming 2006: 84) But in 1971, Ladislav Zgusta had his monograph of Manual of Lexicography published, which is generally regarded as the starting point of lexicography as a relatively independent discipline. In 1989, Franz Josef Hausmann et al compiled the dictionary of An International Encyclopedia 
of Lexicography, which meant that lexicography had made a great leap forward. In 2001, R.R.K. Hartmann and Gregory James published their Dictionary of Lexicography, which elucidated from the historical perspective that lexicography is not a dependency on linguistics but an independent sui generis, liquidating various vague understandings of the disciplinary nature of lexicography, putting forward new ideas about the classification of dictionaries, emphasizing the participation of the users in the dictionary compilation, and illustrating the influence of the publishing media and carriers of dictionaries on the development of the dictionary. Meanwhile, Dictionary of Lexicography has played an active role in normalizing the lexicographical terms.

LÜ Shu-xiang's Modern Chinese Dictionary pioneered the stylistic layout of Chinese dictionaries, which set a good model for the later dictionaries. The Chinese academia of lexicography began to discuss the nature and the theoretical framework of the lexicography as an independent discipline from the end of 1970s. The periodical of Lexicographical Studies issued some important papers about lexicography, e.g. YANG Zu-xi's "Preliminary Discussion of Lexicography" (1979) and "Preliminary Probe into the Basic Problems of Lexicography" (1988), and HUANG Jian-hua's “On Lexicography" (1983, 1985). What's more, a group of lexicographical monographs were published. In 1982, HU Ming-yang had his A Survey of Lexicography published by the People's University of China Press. In 1987 , HUANG Jian-hua's Studies in Lexicography was published by Shanghai Dictionary Press. These two books set the foundation for the lexicographical studies in China. In 1992, Xuelin Press published YANG Zu-xi and XU Qing-kai's Dictionary of Lexicography, in which the authors discussed the dictionary in terms of such aspects as the history, typology, function, and so on, promoting the development of lexicography and normalizing the lexicographical terms. The $20^{\text {th }}$ century boasts its importance in the development of lexicography, home and abroad. In terms of the case in China alone, YONG He-ming wrote:

The lexicographical theories of the $20^{\text {th }}$-century China had developed from a shallow state to a deep one, from the sporadic to the systematic, from the empirical to the theoretical, getting larger and larger in scale, and deeper and deeper in depth. During the $20^{\text {th }}$ century in the mainland of China 7357 academic papers on lexicography were published. In the last two decades of the $20^{\text {th }}$ century the Chinese lexicography got its summit, and stepped into the $21^{\text {st }}$ century with a forceful momentum. (YONG He-ming 2006: 572)

Against the academic background of interdisciplinary studies, lexicography has been influenced by some other disciplines, and it has in turn influenced the other disciplines. The mutual influence will give an endless impetus to the development of lexicography. The development of the other disciplines can also absorb some inspiration from lexicography. The real meaning of lexicography lies in its study of the dictionary compilation and use to improve the quality of the dictionaries, making the metalexicography and dictionary practice fused into a systematic and scientific organic body. And "the role of the metalexicographer is neither merely to describe nor idly to criticize; it is to help the lexicographer put current practice into perspective, and eventually participate in the improvement of dictionaries." (Béjoint 2002: 5)

\section{B. Some Characteristics of Lexicography}

\section{i) Multidisciplinary Integration}

Hartmann and James once wrote:

Lexicography, often misconceived as a branch of linguistics, is sui generis, a field whose endeavors are informed by the theories and practices of information science, literature, publishing, philosophy, and historical, comparative and applied linguistics. Sister disciplines, such as terminology, lexicology, encyclopedia work, bibliography, terminography, indexing, information technology, librarianship, media studies, translation and teaching, as well as the neighboring disciplines of history, education and anthropology, provide the wider setting within which lexicographers have defined and developed their field. (Hartmann \& James 2000: F16)

With the development of the lexicography, people are now well aware of that it can not further its theory and practice without the relevant disciplines. But we know that for a long time in the past the dictionary research had just taken the dictionary-making as a kind of pure linguistic behavior, which was divorced from the other disciplines. The compilation of a dictionary was merely dependent upon the compilers' philological awareness. The relationship between the dictionary and social culture was often ignored. The role of the dictionary in the fields of culture and society was not paid enough attention to. The chief function of the dictionary was generally considered to record, normalize, or spread the language or a particular science. Historically, in the past the awareness of interdisciplinary studies could not creep into the minds of the dictionary researchers, as the physical and cultural conditions were relatively poor. In addition, the interdisciplinary study of a particular subject was really rare in the past, so people had no inspiration to deal with the dictionary with an integrative perspective.

Historically speaking, no dictionary is monotonous in the coverage of its contents. In this regard, YONG He-ming remarked:

The range covered by the contents of the early dictionaries went beyond the definitions of the words. It almost involved every aspect of the human life, ranging from the usage of words, the diction in poetry, vernacular vocabulary, technical vocabulary, law vocabulary, to the other special vocabularies, greatly meeting people's demands in business, law, religion, administration and their everyday life. (YONG He-ming 2006: 37)

The integration of the contents of the dictionary demanded the integration of different compilation theories. Against this background, the modern linguistics triggered the trend. The interdisciplinary characteristic of modern lexicography 
means that lexicography should not be confined to the compilation skills only, but be taken as sui generis involving linguistics, anthropology, sociology, pragmatics, cognitive theory, translatology, computer science and so on. New disciplines are emerging constantly, so the perspectives to study the dictionary are various, and the contents and types of the dictionary are increasingly richened. For example, at the end of 1940s the Americans began to study the machine translation, which during the following decades encouraged many scholars to engage in relevant research. As a consequence, in 1970s people invented the electronic dictionary, which was based on the achievements made in the machine translation. This can be called the outcome of computer science integrated with lexicography. In China, modern lexicography, as YONG He-ming said:

Not only takes in the essence of the traditional dictionary theories and the foreign linguistics, but also the nutrients from the contemporary Chinese linguistics, psychology, pedagogics, communication studies, cognitive theories, information science, computer science, and the social and natural sciences, broadening the research fields of lexicography. The combination between lexicography and the neighboring disciplines is increasingly strengthened, which has produced many branch disciplines, practical or theoretical, e.g. Communicative Lexicography, Cognitive Lexicography, Computer Lexicography, and so on. (YONG He-ming 2006: 605)

Integrating the lexicography and translatology, the translatological dictionary, a kind of specialized dictionary, has emerged as a new academic field in China. The production of the translatological dictionaries (TD) is a logical outcome of the interdisciplinary studies. Nobody can say that there will be no new kinds of dictionaries in the future. Historically speaking, we can say that the development of lexicography lies in its integration with and constant reference to the neighboring disciplines.

\section{ii) Open Perspective}

The compilation skills of the dictionary, the matters covered by the dictionary, and the presentation media of the dictionary are always in evolution, so no one can put a full stop to them. And many other aspects about dictionary will also change with the time passing. Therefore, the perspectives in the study of the dictionary must be open.

The presentation media are in evolution. The changes of the publishing media or carriers of the dictionary have changed, to some extent, people's concept about the dictionary-making and dictionary research. In general, the dictionary is taken as a kind of reference book, which collects the words, deals with the words respectively and organizes the words in a certain order. But how to collect the words, deal with the words, and organize the words is, to some extent, dependent upon the objective physical conditions, which may determine the characteristics of the dictionary. WEI Xiang-qing et al described:

Since the ancient Chinese invented the paper and printing, the publishing media and carriers of the dictionary have experienced about four historical phases. The first phase refers to the handwork tradition of the compilation, which is characterized by the "hand" and "paper"; the second phase refers to printing period, which is characterized by the "fire" and "lead"; the third phase refers to the times of computer, which is characterized by the "light" and "electricity"; the fourth phase refers to the new era of network, which is characterized by the "internet". Every improvement of the media and carriers will always facilitate the spread of the dictionary culture, especially in its breadth and depth. (WEI Xiang-qing et al 2001: 56)

The application of the computer takes a tremendous change to the dictionary compilation and research, which dramatically influences every phase of the dictionary-making. Different from the paper dictionaries, the electronic dictionaries are characterized by the integration of the multi-media and the super interlinkage techniques, making the dictionaries rich in sound, color, picture and the approaches of wording. The on-line dictionaries, which are made up by the language of HTML, take the network as the presentation medium, and they are characterized by the abundant resources, user-friendliness, convenient retrieval, and interactive operation.

WEI Xiang-qing et al argued:

The evolution of the media and carriers of the dictionary not only exerts revolutionary influence on every phase of the compilation and publication of the dictionaries, but also plays an important role in changing people's concept on the reference approach of the dictionary, which have produced new problems, and developed some new research areas, thus enriching the contents of lexicography. (ibid)

The progress of the presentation media of the dictionary does not necessarily mean that the latest medium is the best for all kinds of dictionaries. The paper dictionaries, the electronic dictionaries, and the on-line dictionaries can go forward abreast, as none of them can completely replace the others in certain aspects. Each kind of the dictionary has its strongpoints and shortcomings. They can be mutually complementary in functions. With the development of science and technology, the presentation methods of the dictionary will be doomed to renovate, so it must be open for the researchers to deal with the presentation media or carriers of the dictionaries.

The compilation method is developing all the time. As for the traditional dictionaries, the mountainous materials were often collected by hand, making the dictionary-making a kind of drudgery. With the computer used in the compilation, the compilation methods have been greatly changed. YONG He-ming pointed out:

The dictionary culture of the world has realized the second substantial leap forward with the application of the computer techniques and corpus in the compilation of dictionaries. The electronic technique has penetrated into every inner step of the dictionary compilation and research. It is more direct and efficient than the printing, which can only improve the outer conditions of the dictionary-making, in improving the compilation efficiency, the scientificalness and 
normalization of the dictionaries and their compilation. This can push the dictionary culture of the world to a higher status. (YONG He-ming 2006: 66)

At the annual conference of the third session of the Chinese Association for Lexicography, WANG Yao-nan said that the corpus building and the application of electronic techniques turned to be one of the important agenda at the lexicographical conferences, which had produced an active effect. To apply the electronic corpus to the dictionary theory and practice has proved a trend of lexicography. For example, by using the corpus technique, the Collins has published such dictionaries as Collins COBUILD Dictionary of the English Language, Collins COBUILD Essential Dictionary, Collins COBUILD Learner's Dictionary, Collins COBUILD English Learner's Dictionary, Collins COBUILD Student's Dictionary, International Edition, Collins COBUILD Dictionary of Idioms, and Collins COBUILD Dictionary of Phrasal Verbs. The role of the computer is so important in the contemporary dictionary-making that some lexicographers even put forward a new interdisciplinary subject of Computational Lexicography, which takes linguistics and lexicography as the theoretical basis, and the computer science, computer linguistics, and the corpus linguistics as the technical basis. In the future, the techniques of the dictionary-making borrowed from the computer science and other relevant advanced sciences will be unlimited.

The subjects covered by the dictionary will be unlimited. At the early stage, lexicography was considered as a branch of linguistics, as it directly adopted many theories of linguistics. We may even say there would no lexicography without linguistics. Today, many linguistic theories are still very useful to lexicography. In the West, linguistic theories began to be used in the compilation of dictionaries in the $19^{\text {th }}$ century, but it is in the $20^{\text {th }}$ century that the modern linguistic achievements began to be used in the compilation of dictionaries. YONG He-ming remarked, "From the middle period of the $20^{\text {th }}$ century, the lexicographical academia have theoretically and systematically probed into the application of linguistic studies in the lexicographical theories and practice." (YONG He-ming 2006: 594) For example, CHEN Chu-xiang's "Comparative Linguistics and Bilingual Lexicography”(1992), ZHANG Yi-hua and HUANG Jian-hua's "Effects of Linguistic Theories upon Lexical Definitions" (2000), HU Tao's “On Linguistics and Dictionary-making" (2003) and so on discussed the close relationship between linguistics and lexicography from different perspectives. In addition, in 2004 Shanghai Dictionary Press published WANG Fu-fang's monograph of Current Linguistics and Lexicographic Innovation, which further talked about the various relations between the dictionary-making and linguistics.

Interdisciplinary study of lexicography has been always on the plateau since the end of the $20^{\text {th }}$ century. Let's take the case in China for example. ZHANG Bo-ran's monograph of Essays on Bilingual Lexicographical Studies (2001) talks about the research and compilation of bilingual dictionaries from the perspectives of cognitive linguistics, prototype theory, cultural studies, philosophical theories, and corpus research. LI Ming and ZHOU Jing-hua's monograph of $A n$ Introduction to Bilingual Lexicography (2002) involves the application of corpus in the compilation of bilingual dictionaries. Integrating the communication theories and social culture, YONG He-ming's Lexicography from a Communicative Perspective (2003) puts the users into the framework of communicative theories about dictionaries, probing into the lexicographical problems from an integrative perspective. The content of ZENG Dong-jing's Studies of Bilingual Dictionaries (2003) covers a wide range of topics: the history of bilingual dictionary, compilation of the bilingual dictionary, definition and citation, corpus, culture, pragmatics, lexis, translation, dictionary criticism, readers and dictionaries, and so on. In her book of Studies of the Translated Meaning of Bilingual Dictionaries (2005), WEI Xiang-qing probes into the translated meaning of the bilingual dictionaries by integrating the studies of semantics, bilingual lexicography, translation studies, and receptive reflection theory. In 2008, Sun Ying-chun had his monograph of On Translation and compilation of Scientific Dictionaries published, in which the author discusses the translation and compilation of the scientific dictionaries in a scientific, systematic way by the integration of lexicography and translatology. The list of the various perspectives to deal with the dictionaries, I think, will be endless.

In 2005, the Chinese Association for Lexicography held a conference in Guangzhou, at which Huang Jianhua made a statement like this: with the application of the new linguistic achievements and computer techniques to the dictionary research and making, the compilation, publication, and research of Chinese bilingual dictionaries would certainly present an interdisciplinary, multi-perspective, and multi-dimensional character. His words have now come true. Nowadays, the translatological dictionaries (TD) are a kind of new research subject both to translatology and lexicography.

\section{iii) Practice Orientation}

The dictionary practice or making is very important to lexicography, as it determines the existent meaning of the dictionary theories. All the dictionary theories should aim at improving the quality of the dictionaries, and making the dictionaries practical. YONG He-ming described:

The dictionaries are getting more and more practical, and experts from various fields are increasingly joining in the dictionary compilation and even in the dictionary research. For example, the famous scientists QIAN Xue-sen, YU Guang-yuan, and so on joined in the entry-writing of the large-size dictionaries, and time after time wrote articles expressing their original opinions about the dictionary compilation. (YONG He-ming 2006: 579-580)

To the common people, the dictionary is a good friend in their study and lives. People have well understood the functions of the dictionary to spread knowledge, to instruct, and to solve the problems. In the $21^{\text {st }}$ century, people will have a higher expectation on the dictionary in terms of its variety, quality, typology, and presentation means. There will 
be a better market for the dictionaries, and the dictionaries will be doomed to be diversified. XIA Nan-qiang remarked:

The dictionary is a kind of cultural commodity, and its compilation is preconditioned by the users' needs. Due to the division of the social work, there have formed various user groups. Their demands for the cultural knowledge naturally differ from one another. To study the users and to satisfy their tastes undoubtedly constitute an important aspect in the compilation and publication of the dictionaries. (XIA Nan-qiang 2003: 16)

The user perspective is one of the important factors to make the dictionaries practical. The purpose of the compilers, the function of the dictionaries, and the need of the users should be connected closely. Different users may need different types of dictionaries, and the function of the dictionaries lies in the service of different users.

The practice-oriented characteristic of the dictionaries can also lie in the normalization of the dictionary research, especially the lexicographical terms. For a long time, the normalization of the lexicographical terms has been in a kind of spontaneous state. Sometimes people adhere to the precedent terms, and sometimes they formulate new ones. The development of dictionary research and practice makes it necessary to normalize the lexicographical terms. By the reference to the ISO 19751-1973 "dictionary compilation codes", in 1989 the Chinese State Bureau of Technical Supervision approved the "dictionary compilation codes" of GB 11617-89 as a national standard, and from the following year it came into force. In addition, in 1992 the Chinese Association for Lexicography approved the "basic terms for dictionary compilation" as an international standard. These standards have played a very important role in normalizing the lexicographical terms.

The specialized dictionaries have much clearer purposes in directing the social practice. The richer the social practice is, the richer the specialized dictionaries will be. In terms of the importance of the specialized dictionaries, XU Shi-yi argued:

The specialized dictionaries are important tools to consult the scientific and cultural knowledge. The specialized dictionaries are very rich in their types, involving almost all the scientific and cultural fields. A specialized dictionary of a discipline, especially of a big discipline tends to reflect the level of scientific research within the discipline. The scientific level of a country can be seen, to a certain extent, from the development of its specialized dictionaries. (XU Shi-yi 1995: 97)

In addition, the dictionaries also play an important role in the cultural communication. The intercultural communication between China and the foreign countries is becoming more and more frequent. Against this background, it is necessary for China to know about the outside world, and the outside world to know about China. The various dictionaries can facilitate the cultural communication, and bridge the cultural gaps. To make a comprehensive survey of the development course of the Chinese dictionaries, we can find out the close relationship between the dictionary-making and social demands. The history of the dictionary is just a history to serve the society.

\section{TRAnSLATOlOGiCAl Dictionaries}

The multidisciplinary integration of lexicography elucidates that modern dictionary making or research can not be dealt with from any single discipline. The interdisciplinary study of the dictionary suits with the trend of modern learning. The translatological dictionaries (TD) are just the outcome of the integration of lexicography and translatology. As for the "open perspectives", I mainly think that the perspectives to study the dictionaries should be various and endless, so the TD, a new dictionary type, must be reasonable in their existence. By "practice orientation", I mean that all the dictionaries should be practice-oriented, as they are used either to describe people's practice or to normalize people's practice. The naissance of the TD can meet people's demands in their translation research or practice.

Comparatively, the TD are much younger, with a short history of more than two decades. Therefore, the study of translatological dictionaries (STD) is too young to arouse enough attention from the scholars. As a matter of fact, the translatological dictionaries are produced from the integration of translation theories and lexicographical ones. Theoretically, the emergence of some influential translatological dictionaries can well represent the maturity and independence of translation studies as a discipline. The year of 1988 met the first publication of a Chinese translatological dictionary-A Dictionary of Chinese Translators. Up to now, there have appeared about 20 translatological dictionaries in the world, among which 4 are generally considered as the most important in China. These four dictionaries are A Companion for Chinese Translators (1997) mainly compiled by LIN Huang-tian, Aspects of Translation (1999) by SUN Ying-chun, Dictionary of Translation Studies (1997) by Mark Shuttleworth \& Moira Cowie and Routledge Encyclopedia of Translation Studies (1998) by Mona Baker.

To probe into the compilation theories of the TD, in 2001, SUN Ying-chun wrote an article of "A Brief Exposition on the Compilation of Comprehensive Dictionaries of Translatology" published by Shandong Foreign Languages Journal, which pioneered the STD. From then on, many Chinese scholars have gone in for the STD, dealing with the translatological dictionaries in terms of the nature, methodology, typology, structure, function, criticism and so forth. The STD aims at systematically dealing with the translatological knowledge by means of the form of the dictionary. In fact, we can conduct the translation studies from various perspectives, since translatology is open in nature. Gideon Toury once said:

Each of these question areas is legitimate, as well as interesting; and each one of us may choose an area to his or her own liking. Still, it should be realized that they belong to different domains of Translation Studies, and it simply won't do to mix them all in one neutral, or neutralizing bag. (Anderman \& Rogers 2006: 18) 
As a consequence, there should be various schools of scholars in the translation studies, some turning to descriptive studies, some explanatory, and some normative. The STD should be regarded as one of these various schools. The translatological dictionaries are precious resources for the theoretical research of the TD. Professor SUN Ying-chun's article of On the Compilation of Comprehensive Translatological Dictionaries triggered the study of the TD in China. From then on, the academic study of the TD has met its five national conferences: Yantai conference in 2002, Weihai conference in 2004, Shanghai conference in 2005, Dalian conference in 2007, and Weihai conference in 2009. In September 2006 A Doctoral Anthology of Translatological Dictionary Studies, which was chiefly edited by SUN Ying-chun and assisted by HUANG Xi-ling and ZHAO Wei, was published by Tianjin Education Press. In April 2007 Professor ZENG Dong-jing's monograph Theoretical Research of the Compilation of Translatological Dictionaries was published by Shanghai University Press. In 2009, SUN Ying-chun's monograph On Translatological Dictionaries is published by Shanghai Foreign Language Education Press, in which the author mainly discusses the TD in terms of its nature, function, typology, compilation principle, macrostructure, microstructure and so on, and discusses the STD in terms of its the research object and structure, disciplinary location, value and evaluation, methodology and so on. With the deepening in the STD, the name and nature of the TD have been gradually established, and the knowledge of the TD has been improved from the perception to reason.

\section{REFERENCES}

[1] Anderman, G. \& M. Rogers. (2006). Translation Today: Trends and Perspectives. Beijing: Foreign Language Teaching and Research Press.

[2] Baker, M. (2004). Routledge Encyclopedia of Translation Studies. Shanghai: Shanghai Foreign Language Education Press.

[3] Béjoint, Henri. (2002). Modern Lexicography: An Introduction. Beijing: Foreign Language Teaching and Research Press.

[4] Bo-ran ZHANG, Xiang-qing WEI. (2001). Essays on Bilingual Lexicographical Studies. Nanjing: Jiangsu Education Press.

[5] Dong-jing ZENG. (2003). Studies of Bilingual Dictionaries. Shanghai: Shanghai Foreign Language Education Press.

[6] Dong-jing ZENG. (2007). Theoretical Research of the Compilation of Translatological Dictionaries. Shanghai: Shanghai University Press.

[7] Er-gang LI. (2002). Introduction to Modern Lexicography. Shanghai: Chinese Dictionary Press.

[8] Hartmann, R.R.K. (2005). Teaching and Researching Lexicography. Beijing: Foreign Language Teaching and Research Press.

[9] Hartmann, R.R.K. \& G. James. (2000). Dictionary of Lexicography. Beijing: Foreign Language Teaching and Research Press.

[10] He-ming YONG. (2003). Lexicography from a Communicative Perspective. Shanghai: Shanghai Foreign Language Education Press.

[11] He-ming YONG et al. (2006). On the History of Chinese Dictionary. Beijing: Zhonghua Book Company Press.

[12] Huang-tian Lin. (1997). A Companion for Chinese Translators. Wuhan: Hubei Education Press.

[13] Jian-hua HUANG. (2001). On Dictionary. Shanghai: Shanghai Lexicographical Publishing House.

[14] Ming-yang HU et al. (1982). Introduction to Lexicography. Beijing: People's University of China Press.

[15] Ming LI, Jing-hua ZHOU. (2002). An Introduction to Bilingual Lexicography. Shanghai: Shanghai Foreign Language Education Press.

[16] Nan-qiang XIA. (2003). Dictionary Compilation Should Advance With the Times. Editor's Friend 3.

[17] Shuttleworth, M. \& M. Cowie. (2004). Dictionary of Translation Studies. Shanghai: Shanghai Foreign Language Education Press.

[18] Shi-yi XU. (1995). Social Demands and the Dictionary Compilation. Lexicographical Studies 4.

[19] Xiang-qing WEI et al. (2001). New Horizon of Lexicographical Studies in the New Century. Foreign Languages and Their Teaching 4.

[20] Xiang-qing WEI.(2005). Definition Research of the Bilingual Dictionaries. Shanghai: Shanghai Translation Publishing House.

[21] Ying-chun SUN. (1999). Aspects of Translation. Beijing: Chinese Foreign Languages Press.

[22] Ying-chun SUN. (2001). A Brief Exposition on the Compilation of Comprehensive Dictionaries of Translatology. Shandong Foreign Languages Teaching 1.

[23] Ying-chun SUN. (2005). 2004 Anthology of Translatological Dictionary and Translation Theory Studies. Tianjin: Tianjin Education Press.

[24] Ying-chun SUN. (2006). A Doctoral Anthology of Translatological Dictionary Studies. Tianjin: Tianjin Education Press.

[25] Ying-chun SUN. (2008). On Translation and Compilation of Scientific Dictionaries. Beijing: China Translation \& Publishing Corporation.

[26] Ying-chun SUN. (2009). On Translatological Dictionaries. Shanghai: Shanghai Foreign Language Education Press.

[27] Zu-xi YANG, Qing-kai XU. (1992). Specialized Lexicography. Chengdu: Sichuan Lexicographical Publishing House.

Lei Gao was born in Lianyungang, China in 1975. He is currently a PH.D. candidate in translatology in Shandong University, China. He is currently a lecturer in the School of Foreign Languages, Huaiyin Normal University, Huai'an, China. His research interests include translation studies and studies of translatological dictionaries. 